Jörg Melzer ${ }^{a, b}$

\title{
Zu Garvelmann F: «Traditionelle Europäische Naturheilkunde - ein eigenständiges Medizinsystem ". Schweiz Z Ganzheitsmed 2013;25: DOI: 10.1159/000351368
}

Der Fortbildungsartikel «Traditionelle Europäische Naturheilkunde ein eigenständiges Medizinsystem» gibt einen essayistischen Einblick in subjektiv ausgewählte historische Aspekte der TEN. Der Schwerpunkt liegt bei historischen Ausführungen vor allem zu antiken und andeutungsweise $\mathrm{zu}$ mittelalterlichen oder neuzeitlichen Medizinkonzepten. Diese historischen Verweise dienen dann dazu, eigene Therapievorstellungen quasi zu belegen.

Leider kommt dabei eine medizinhistorische und notwendige kritische Reflektion aus einer modernen naturheilkundlichen Sicht zu kurz. Diese ist aber notwendig, um die TEN vor dem Vorwurf dogmatischen Festhaltens an traditionellen Theorien zu bewahren, die im historischen Rückblick leicht veraltet erscheinen. Dies schliesst notwendigerweise Fragen nach der Legitimation der nahezu Eins-zueins-Übernahme historischer Theorien ein. Medizinhistorisches sollte in diesem Kontext Ausgangspunkt für Überlegungen zum möglichen oder unmöglichen Transfer historischer Konzepte und Termini in die heutige TEN sein. Hierzu wäre ein Diskurs vor dem Hintergrund unserer heutigen wissenschaftstheoretischen und klinischen Kenntnisse sowie Forschungsergebnisse notwendig. Historisches kann man so im ursprünglichen Kontext würdigen. Durch

\footnotetext{
anstitut für Naturheilkunde,

${ }^{\text {b} K l i n i k ~ f u ̈ r ~ P s y c h i a t r i e ~ u n d ~ P s y c h o t h e r a p i e, ~}$ UniversitätsSpital Zürich, Schweiz
}

kritische Rezeption und Auseinandersetzung mit heutigem Wissen ist dann eine Weiterentwicklung der Theorien der TEN möglich. Solch eine Überprüfung traditioneller Theorien ist ein anspruchsvoller und wichtiger Beitrag zur wissenschaftlichen Etablierung der TEN.

Viele Details im Artikel reizen zum Widerspruch (etwa das Postulieren energetisch-informativer Prinzipien und die Behauptung einer Renaissance der TEN im deutschsprachigen Raum - beides indes ohne Erklärung; die Darstellung der Lehren hippokratischer Ärzte oder Galens als Alleinstellungsmerkmal der Naturheilkunde; die Auffassung, Psychosen oder Neurosen entstünden durch "pathogene Reize»; die Aussage, Humoralpathologie sei ein zentrales Element der heutigen TEN inklusive Antlitz-, Iris-, Zungen-, Pulsdiagnostik oder Harnschau). Dies soll hier im Rahmen der Einladung zu einem Kommentar aber nicht näher erörtert werden. Vielmehr scheint es wichtig, zwei unmittelbar für die praktische ärztliche Arbeit relevante Aspekte aufzugreifen:

- Die Hypothese, die TEN arbeite funktionsorientiert und die Schulmedizin strukturorientiert, kann in der absoluten Formulierung nicht aufrechtgehalten werden. Zumindest müsste auf die Schnittmenge zwischen beiden Bereichen, die funktionellen Störungen, hingewiesen werden. Sie sind im ICD-10 (International Statistical Classification of Diseases and Related Health Problems) als so- matoforme Störungen zusammengefasst und fordern die Praktiker quer durch die medizinischen Fachbereiche (z.B. Innere Medizin inklusive Gastroenterologie und Kardiologie, Gynäkologie, Urologie, Neurologie sowie Rheumatologie) in ihrem differentialdiagnostischen Können. Das mag auch den Aspekt des Status der heutigen Naturheilkunde als der eines medizinischen Querschnittsfachs teilweise erklären. Über somatoforme Störungen könnten auch historische Bezüge zwischen Naturheilkunde und Schulmedizin hergestellt werden: Personell über die naturheilkundlichen Ärzte wie Maximilian Bircher-Benner und Georg Groddeck, inhaltlich über unspezifische Psychotherapieformen und fachlich über die Psychosomatik. Freilich ist heute formell die Psychiatrie und Psychotherapie bzw. je nach Land die Konsiliarund Liaisonpsychiatrie oder die Psychosomatische Medizin für somatoforme Störungen zuständig. Jedoch sind entwicklungsgeschichtlich einige Berührungspunkte vorhanden.

- Die Aussage, dass «eine schulmedizinische Diagnose keine Basis für ein naturheilkundliches Therapiekonzept sein kann», ist absurd und widerspricht dem Berufsethos. Dem muss zum Abwenden von Gefahren für Patienten sowie aus der Sicht der universitären Naturheilkunde energisch widersprochen werden. Mit solchen Bemer-

\section{KARGER}

Fax +4976145207 14
(๑) 2013 S. Karger GmbH, Freiburg

Accessible online at: www.karger.com/sz
Dr. med. Jörg Melzer

Institut für Naturheilkunde, UniversitätsSpital Zürich

Rämistrasse 100, 8091 Zürich, Schweiz

joerg.melzer@usz.ch 
kungen oder Einstellungen ruft man zu Recht Kritiker auf den Platz, und zwar - wenn man die dichotome Unterscheidung bemühen will - sowohl Naturheilkundler als auch Schulmediziner. Denn qualitativ hochstehende und fachgerechte Behandlung setzt wissenschaftlich akzeptierte Diagnostik voraus (operationalisierbar, objektivierbar, reproduzierbar, valide, reliable). Dies Patienten durch ein Beharren auf Tradition vorzuenthalten, ist nicht zu rechtfertigen. Es ist enttäuschend, dass eine Grundlage für ein eigenes Diagnosesystem einer modernen TEN nicht genannt wird. Dies wäre angesichts des internationalen Standards akzeptierter diagnostischer Klassifi- kationssysteme wie dem ICD-10 oder DSM-IV (Diagnostic and Statistical Manual of Mental Disorders) als Diskussionsgrundlage aber nötig.

Fazit

So wird insgesamt die angekündigte Darstellung eines «sich lebendig weiterentwickelnden Medizinsystems» leider nicht erfüllt. Allerdings zeigt dieser Artikel die enorme Variationsbreite in der Naturheilkunde - zwischen Praktikern, die aufgrund historischer Theorien eigene Vorstellungen und eventuell Konzepte entwickeln, Praktikern, die ihre eigene Empirie mit Tradition und Aspekten der evi- denzbasierten Medizin (EBM) verbinden, und universitären Vertretern der Naturheilkunde, die methodisch der EBM verpflichtet sind, diese mit der Empirie abgleichen und von dort gelegentliche Exkurse in die Tradition unternehmen. Alle drei Gruppen prägen das heutige Bild der Naturheilkunde in der Gesellschaft. Diese Variationsbreite ist sicher aus der zu lange fehlenden universitären Anknüpfung des Fachs erklärbar. Vonseiten der Evidenz wurde viel erreicht. Die Diskussion unter den divergierenden Gruppierungen der Naturheilkundler ist jedoch noch längst nicht abgeschlossen. Hat sie überhaupt begonnen? Der Artikel könnte ein Beginn sein. 